\title{
ORIGINAL
}

\section{EXPOSICIÓN A CARGA FÍSICA EN EL TRABAJO POR OCUPACIÓN: UNA EXPLOTACIÓN DE LOS DATOS EN MATRIZ EMPLEO-EXPOSICIÓN ESPAÑOLA ( MATEMESP) (*)}

\author{
$M^{\mathrm{a}}$ Carmen González-Galarzo (1), Ana $\mathrm{M}^{\mathrm{a}}$ García (1,2,3), Rafael Gadea Merino (4), José \\ Miguel Martínez Martínez (1,3), José María Velarde Collado (1).
}

(1) Centro de Investigación en Salud Laboral. Universidad Pompeu Fabra.

(2) Departamento de Medicina Preventiva y Salud Pública. Universidad de Valencia.

(3) Centro de Investigación Biomédica en red de epidemiología y salud pública (CIBERESP).

(4) Conselleria de Sanitat. Generalitat Valenciana.

(*) Finanaciación: Instituto de Salud Carlos III (referencia PI081496), Conselleria de Sanitat de la Generalitat Valenciana (referencia 066/2009). Instituto Nacional de Seguridad e Higiene en el Trabajo y Fundación Prevent.

Los autores declaran no tener ningún conflicto de intereses.

\section{RESUMEN}

Fundamentos: La exposición a riesgos ergonómicos es la principal causa de daños de origen laboral. El objetivo es describir la prevalencia de exposición a carga física por ocupación en población laboral española y su relación con las condiciones de empleo y características sociodemográficas de los trabajadores.

Métodos: Se utilizó la información contenida en la Matriz EmpleoExposición Española (MatEmESp) con datos entre 1997 y 2005. Se describieron las prevalencias de exposición a riesgos ergonómicos, identificándose las ocupaciones con mayores prevalencias y se analizó la relación con las condiciones de empleo y las características sociodemográficas por ocupación mediante coeficientes de correlación de Spearman y diagramas de dispersión.

Resultados: Los movimientos repetidos fueron el riesgo ergonómico declarado con mayor frecuencia (prevalencias en torno al 60\%). Las mayores prevalencias de exposición a posturas forzadas, manipulación de cargas, movimientos repetidos y trabajo sedentario se dieron en "albañiles" $(96 \%)$, en "peones de construcción" $(89 \%)$, en "trabajadores de artes gráficas" (95\%) y "auxiliares administrativos" (98\%), respectivamente. Encontramos una fuerte relación $(\mathrm{p}<0,001)$ entre la prevalencia de exposición a estos cuatro riesgos y la proporción de personas con estudios primarios en la ocupación (correlación - 0,62 en trabajo sedentario y entre 0,59 y 0,66 en el resto). Ocupaciones con alta proporción de mujeres y nivel de estudios universitarios ("enfermeras"), también mostraron prevalencias de exposición elevadas a manipulación de cargas $(>50 \%)$.

Conclusiones: En general, la prevalencia de exposición a riesgos ergonómicos se mantiene muy elevada en el periodo analizado. Las ocupaciones menos cualificadas presentaron habitualmente mayores prevalencias de exposición.

Palabras clave: Exposición laboral. Ocupaciones. Ergonomía. Condiciones de trabajo. Desigualdades.

Correspondencia

M Carmen González Galarzo

Centro de Investigación en Salud Laboral

Parc de Recerca Biomédica de Barcelona

$\mathrm{C} /$ Dr Aiguader, 88

08003 Barcelona.

Correo electrónico: mcarmen.gonzalez@upf.edu

\section{ABSTRACT \\ Exposure to Ergonomic Risk by Occupation in Spanish Working: a Data Exploitation of MatEmESp}

Background: Ergonomic hazards exposure is the main cause of occupational pain. The aim of this paper is to describe the prevalence of exposure to occupational physical load by occupation, in Spanish working population, and its relationship to employment conditions and sociodemographic characteristics of workers.

Methods: We used the information contained in the Spanish JobExposure Matrix (MatEmESp), with data between 1997 and 2005. We describe the prevalence of exposure to ergonomic hazards, we identify the occupations with the highest prevalence of exposure and we analyze its relationship to employment conditions and sociodemographic characteristics by occupation, using Spearman correlation coefficients and Scatter plots.

Results: Repetitive movements are ergonomic hazard most frequently reported (prevalence around $60 \%$ ). The greater prevalence of exposure to awkward postures are given in "Bricklayers" (96\%), to handling loads in "construction labourers" (89\%), to repetitive movements in "graphic arts workers" (95\%) and sedentary work in "administrative assistants" (98\%). We found a strong relationship $(\mathrm{p}<0,001)$ between the prevalence of exposure to the four hazards considered, and the proportion of people with primary education by occupation (correlation coefficients $-0,62$ in sedentary work and between 0,59 and 0,66 in the other). Occupations with a high proportion of women and with a university studies levels ("nurses"), also has frequent exposure to handling loads $(>50 \%)$.

Conclusions: In general, the prevalence of exposure to ergonomic hazards remains high in the period analyzed. Less qualified occupations usually have a higher prevalence of exposure.

Keywords: Occupational exposure. Occupations. Ergonomics. Working conditions. Health inequalities. 


\section{INTRODUCCIÓN}

Las matrices empleo-exposición han sido ampliamente utilizadas en epidemiología ocupacional ${ }^{1}$. Básicamente, una matriz empleo-exposición (MEE) es un sistema de información o base de datos que reúne la información disponible acerca de agentes o exposiciones laborales que están presentes en cada ocupación o puesto de trabajo para un determinado sector, empresa o ámbito territorial, normalmente referida a un momento o período determinado. Por tanto, una matriz empleo-exposición recopila información disponible de distintas fuentes y en distintos períodos y homogeneiza el uso de esa información reuniendo todos los datos a través de un eje común, la ocupación, facilitando así análisis simultáneos considerando distintos agentes de exposición y circunstancias asociadas a cada ocupación. En España se han llevado a cabo con cierta frecuencia estudios que han utilizado la información de matrices empleo-exposición elaboradas en otros países $^{2-4}$, pero hasta el momento no se disponía de una MEE específica para población laboral española. El proyecto MatEmESp (Matriz Empleo-Exposición Española) se inició en 2009 con el objetivo de construir una matriz empleo exposición específica para población laboral española que cubriera el período 1996-2005. MatEmESp es una matriz general con el objetivo de cubrir a toda la población laboral y de incluir información para todas las categorías de riesgos en el trabajo ${ }^{5}$ que, básicamente, organiza la información en dos ejes, uno de ocupaciones y otro de agentes. Para la construcción del eje de ocupaciones se siguió la Clasificación Nacional de Ocupaciones 1994 (CNO-94) ${ }^{6}$ con dos, tres o cuatro dígitos según la información disponible. El eje de agentes contiene las estimaciones de prevalencia e intensidad para exposiciones o factores laborales agrupados en cinco categorías: agentes de higiene, de seguridad, ergonómicos y psicosociales y condiciones de empleo. MatEmESp también contiene información sobre las características sociodemográficas de los traba- jadores en cada ocupación y estimaciones de la exposición por sexo y categorías de edad, en las ocupaciones y agentes donde las fuentes de información lo han permitido. Se puede obtener más detalle acerca de las fuentes y métodos seguidos en la construcción de la matriz en una publicación previa ${ }^{7}$ y en la página web desde la que se accede a los contenidos de MatEmEsp (www.matemesp.org).

La inclusión de información sobre la exposición laboral a riesgos ergonómicos y sobre las condiciones de empleo y características sociodemográficas de los trabajadores en las matrices empleo-exposición no es habitual, aunque hay algunas excepciones. Por ejemplo, la matriz empleo-exposición finlandesa (FINJEM) ${ }^{8}$, una matriz general para población trabajadora, incluye estimaciones de exposición a algunos riesgos ergonómicos, así como indicadores sobre condiciones de empleo y, en algunos casos, diferentes estimadores de exposición para hombres y mujeres. Otras MEE específicas (creadas exclusivamente para la evaluación de algún tipo concreto de exposición o de daños o referidas exclusivamente a algún sector de actividad u ocupación) incluyen también información referida a exposición a carga física en el trabajo y/o condiciones de empleo y características de los trabajadores. Es el caso de una MEE centrada en factores de riesgo laboral para el síndrome de túnel carpiano ${ }^{9}$ y otra para la neuropatía cubital ${ }^{10}$, de otra matriz orientada hacia aspectos relacionados con la organización de trabajo ${ }^{11}$, incluyendo también indicadores de exposición a carga física y de algunas MEE referidas a ocupaciones o sectores de actividad específicos, por ejemplo conserjes escolares ${ }^{12}$, personal sanitario $^{13} \mathrm{o}$ trabajadores en la industria del gas ${ }^{14}$. En la elaboración de todas estas MEE encontramos metodologías similares: los datos suelen proceder de encuestas rutinarias a nivel nacional (como el National Health Interview Survey o la base de datos $\mathrm{O}^{\prime} \mathrm{NET}^{15}$ de Estados Unidos ${ }^{9}, 13$, o el Survey of Living Conditions de Suecia ${ }^{11}$, de cues- 
tionarios específicos a los trabajadores ${ }^{14}$ y/o valoración de expertos ${ }^{10,13}$.

Las MEE se han utilizado principalmente en investigación etiológica en el campo de la epidemiología laboral, en la búsqueda de asociaciones entre exposiciones laborales y problemas de salud en los trabajadores. Pero también se ha señalado su utilidad para la planificación y seguimiento de las acciones de prevención de riesgos laborales en un ámbito específico, para orientar la vigilancia de la salud de los trabajadores y/o como herramienta de apoyo para los profesionales sanitarios en la identificación de problemas de salud laboral ${ }^{7,8}$. Adicionalmente, la explotación descriptiva de los datos contenidos en una MEE puede ser de utilidad a la hora de identificar a los colectivos de trabajadores más expuestos y más vulnerables, facilitando la priorización de políticas y programas preventivos. En este último sentido, las MEE pueden resultar de interés en la investigación sobre desigualdades en salud en la población trabajadora.

El objetivo de este trabajo fue describir la prevalencia de exposición a los principales riesgos ergonómicos por ocupación y su relación con factores sociodemográficos y condiciones de empleo de los trabajadores, basándonos en la información contenida en MatEmESp, la primera matriz empleoexposición para población trabajadora en España.

\section{MATERIAL Y MÉTODOS}

En MatEmESp se incluyeron estimaciones de la exposición por ocupación a los siguientes riesgos ergonómicos: posturas forzadas, manipulación de cargas, esfuerzo físico, movimientos repetidos, trabajo sedentario, trabajo de pie, vibraciones y utilización de pantallas de visualización de datos. Los estimadores disponibles para estos riesgos fueron el porcentaje de trabajadores expuestos y la intensidad de la exposición (media, mediana y desviación típica) en una escala de 1-2 o 1-4 en el caso de posturas forzadas. Las fuentes de información fueron las encuestas nacionales de condiciones de trabajo (ENCT) de 1997, 1999 y $2003^{16}$, la I Encuesta Catalana de Condiciones de Trabajo (ECCT) de $2005^{17}$ y la Encuesta Nacional de Condiciones de Trabajo en el Sector Agropecuario (ENCTA) de $2007^{18}$, considerándose sólo las ocupaciones con un número de entrevistados en cada encuesta igual o superior a 20. La descripción de estos riesgos y los criterios de evaluación de la exposición que se siguieron en MatEmESp para obtener los correspondientes estimadores de exposición se muestran en las tablas 1 y 2 .

MatEmESp también contiene información sobre las condiciones de empleo en cada ocupación: proporción de ocupados según situación profesional (empleador, autónomo, asalariado o ayuda familiar), tipo de contrato (temporal), tipo de jornada (tiempo parcial) y tiempo de trabajo (fin de semana, nocturno, turnos) y estimadores de tendencia central (media y mediana) y dispersión (desviación típica) para horas de trabajo semanales y salario base (neto y bruto). Además, describe en cada ocupación la distribución de trabajadores (número y porcentaje) por grupos de edad (menos de 31 años, 31-45 años y más de 45 años), sexo, nivel de estudios (sin estudios, primarios, secundarios, universitarios), país de origen (España, otros), actividad económica (CNAE-93 con códigos a 1 dígito) ${ }^{19}$ y clase social ocupacional (categorías $\mathrm{I}-\mathrm{V})^{20}$. La información sobre condiciones de empleo y características sociodemográficas por ocupación de los trabajadores procede de la Encuesta de Población Activa (EPA) de 1996, 2000 y 2005 y la Encuesta de Estructura Salarial (EES) de 1995, 2000 y 2006, ambas disponibles en las bases de datos del Instituto Nacional de Estadística (www.ine.es).

Toda la información se relacionó con las ocupaciones con dos y tres dígitos de la 
Tabla 1

\section{Definición de la exposición a riesgos ergonómicos en MatEmEsp Encuestas nacionales de condiciones de trabajo (ENCT)}

\begin{tabular}{|c|c|c|}
\hline AGENTE & DEFINICIÓN DEL AGENTE & UNIDAD DE MEDIDA \\
\hline Posturas forzadas & $\begin{array}{l}\text { Realizar posturas de trabajo forzadas. La evaluación se rea- } \\
\text { liza en base a la respuesta a } 4 \text { ítems: } 1 \text {. Su trabajo le obliga a: } \\
\text { Permanecer en posturas dolorosas o fatigantes, más de } 1 / 4 \\
\text { de la jornada. } 2 \text {. En su trabajo es frecuente: Tener que alcan- } \\
\text { zar herramientas, elementos u objetos de trabajo situados } \\
\text { muy altos o muy bajos que obliguen a estirar mucho el bra- } \\
\text { zo. } 3 \text {. En su trabajo es frecuente: Trabajar en zonas de muy } \\
\text { difícil acceso para las manos. } 4 \text {. Postura habitual de trabajo: } \\
\text { Trabajar de pie con las rodillas ligeramente flexionadas. Ca- } \\
\text { tegorías de respuesta (niveles de } 0 \text { a } 4 \text { según son afirmativas } \\
\text { las preguntas anteriores). } \alpha \text { de Cronbach de la escala: } 0,54 \text {. } \\
\text { (III-ENCT); } 0,34 \text { (IV-ENCT); } 0,50 \text { (V-ENCT). }\end{array}$ & $\begin{array}{l}\text { Nivel [puntuación media/me- } \\
\text { diana/ desviación típica de una } \\
\text { escala de (1-4)]; prevalencia } \\
\text { [expuestos a cualquier nivel de } \\
\text { exposición }(\%) \text { ]. }\end{array}$ \\
\hline $\begin{array}{l}\text { Manipulación } \\
\text { de cargas }\end{array}$ & $\begin{array}{l}\text { Manipulación manual de cargas. La evaluación se realiza en } \\
\text { base a la respuesta a un ítem: Su trabajo le obliga a: Levan- } \\
\text { tar o desplazar cargas pesadas. Categorías de respuesta: } \\
\text { Nunca, } 0 \text { / A veces, } 1 \text { / Siempre o muchas veces, } 2 \text {. }\end{array}$ & $\begin{array}{l}\text { Nivel [puntuación media/me- } \\
\text { diana/ desviación típica de una } \\
\text { escala de (1-2)]; prevalencia } \\
\text { [expuestos a cualquier nivel de } \\
\text { exposición }(\%) \text { ] }\end{array}$ \\
\hline $\begin{array}{l}\text { Esfuerzo } \\
\text { físico percibido }\end{array}$ & $\begin{array}{l}\text { Percepción del esfuerzo físico realizado. La evaluación se } \\
\text { realiza en base a la respuesta a un ítem: Su trabajo le obliga } \\
\text { a: Realizar una fuerza importante. Categorías de respuesta: } \\
\text { Nunca, } 0 \text { / A veces, } 1 \text { / Siempre o muchas veces, } 2 \text {. }\end{array}$ & $\begin{array}{l}\text { Nivel [puntuación media/me- } \\
\text { diana/ desviación típica de una } \\
\text { escala de }(1-2) \text { ]; prevalencia } \\
\text { [expuestos a cualquier nivel de } \\
\text { exposición }(\%) \text { ] }\end{array}$ \\
\hline $\begin{array}{l}\text { Movimientos } \\
\text { repetidos }\end{array}$ & $\begin{array}{l}\text { Realizar movimientos repetidos de extremidades superiores } \\
\text { en el trabajo. La evaluación se realiza en base a la respuesta } \\
\text { a un ítem: Su trabajo le obliga a: Realizar movimientos de } \\
\text { manos o brazos muy repetitivos. Categorías de respuesta: } \\
\text { Nunca, } 0 \text { / A veces, } 1 \text { / Siempre o muchas veces, } 2 \text {. }\end{array}$ & $\begin{array}{l}\text { Nivel [puntuación media/me- } \\
\text { diana/ desviación típica de una } \\
\text { escala de (1-2)]; prevalencia } \\
\text { [expuestos a cualquier nivel de } \\
\text { exposición }(\%) \text { ] }\end{array}$ \\
\hline $\begin{array}{l}\text { Trabajo } \\
\text { sedentario }\end{array}$ & $\begin{array}{l}\text { Realizar un trabajo sedentario. La evaluación se realiza en } \\
\text { base a la respuesta a un ítem: Postura habitual de trabajo: } \\
\text { Sentado, sin levantarse casi nunca. Categorías de respuesta: } \\
\text { No, } 0 / \text { Sí, } 1 .\end{array}$ & $\begin{array}{l}\text { Prevalencia [expuestos a cual- } \\
\text { quier nivel de exposición }(\%) \text { ] }\end{array}$ \\
\hline Trabajar de pie & $\begin{array}{l}\text { Trabajar de pie. La evaluación se realiza en base a la res- } \\
\text { puesta a un ítem: Postura habitual de trabajo: De pie sin an- } \\
\text { dar apenas. Categorías de respuesta: No, } 0 / \text { Sí, } 1 .\end{array}$ & $\begin{array}{l}\text { Prevalencia [expuestos a cual- } \\
\text { quier nivel de exposición }(\%) \text { ] }\end{array}$ \\
\hline Vibraciones & $\begin{array}{l}\text { Vibraciones de mano-brazo o cuerpo entero. La evaluación } \\
\text { se realiza en base a la respuesta a un ítem: ¿Tiene Vds. vi- } \\
\text { braciones en su puesto de trabajo? Categorías de respuesta: } \\
\text { No, } 0 \text { / Sí, } 1 .\end{array}$ & $\begin{array}{l}\text { Prevalencia [expuestos a cual- } \\
\text { quier nivel de exposición }(\%) \text { ] }\end{array}$ \\
\hline $\begin{array}{l}\text { Pantallas de } \\
\text { visualización } \\
\text { de datos }\end{array}$ & $\begin{array}{l}\text { Utilización de pantallas de visualización de datos. La eva- } \\
\text { luación se realiza en base a la respuesta a un ítem: Instru- } \\
\text { mento fundamental en su trabajo: Equipos informáticos. Ca- } \\
\text { tegorías de respuesta: No, } 0 \text { / Sí, } 1 \text {. }\end{array}$ & $\begin{array}{l}\text { Prevalencia [expuestos a cual- } \\
\text { quier nivel de exposición }(\%) \text { ] }\end{array}$ \\
\hline
\end{tabular}


Tabla 2

\section{Definición de la exposición a riesgos ergonómicos en MatEmEsp Encuesta Catalana de Condiciones de Trabajo}

\begin{tabular}{|c|c|c|}
\hline AGENTE & DEFINICIÓN DEL AGENTE & UNIDAD DE MEDIDA \\
\hline Posturas forzadas & $\begin{array}{l}\text { Realizar posturas de trabajo forzadas. La evaluación se rea- } \\
\text { liza en base a la respuesta a cuatro ítems: } 1 \text {. Su trabajo im- } \\
\text { plica: Permanecer en posturas dolorosas o fatigantes. } 2 \text {. Al- } \\
\text { canzar herramientas, elementos u objetos de trabajo que } \\
\text { obligan a estirar mucho los brazos. } 3 \text {. Trabajar en zonas de } \\
\text { muy difícil acceso para las manos. } 4 \text {. Trabajar con los brazos } \\
\text { por encima de los hombros. Categorías de respuesta: niveles } \\
\text { de } 0 \text { a } 4 \text { según declaran exposición (a veces, muchas veces o } \\
\text { siempre) en las preguntas anteriores. } \alpha \text { de Cronbach de la es- } \\
\text { cala: } 0,657 \text {. }\end{array}$ & $\begin{array}{l}\text { Nivel [puntuación media/me- } \\
\text { diana/ desviación típica de una } \\
\text { escala de (1-4)]; prevalencia } \\
\text { [expuestos a cualquier nivel de } \\
\text { exposición }(\%) \text { ] }\end{array}$ \\
\hline $\begin{array}{l}\text { Manipulación } \\
\text { de cargas }\end{array}$ & $\begin{array}{l}\text { Manipulación manual de cargas. La evaluación se realiza en } \\
\text { base a la respuesta a un ítem: Su trabajo implica: Levantar o } \\
\text { desplazar cargas pesadas manualmente. Categorías de res- } \\
\text { puesta: Nunca, 0/ A veces, } 1 \text { / Siempre o muchas veces, } 2 \text {. }\end{array}$ & $\begin{array}{l}\text { Nivel [puntuación media/me- } \\
\text { diana/ desviación típica de una } \\
\text { escala de }(1-2) \text { ]; prevalencia } \\
\text { [expuestos a cualquier nivel de } \\
\text { exposición }(\%) \text { ] }\end{array}$ \\
\hline $\begin{array}{l}\text { Movimientos } \\
\text { repetidos }\end{array}$ & $\begin{array}{l}\text { Realizar movimientos repetidos de extremidades superiores } \\
\text { en el trabajo. La evaluación se realiza en base a la respuesta } \\
\text { a un ítem: Su trabajo implica: Realizar movimientos repetiti- } \\
\text { vos de corta duración (de brazos, muñecas y otras articula- } \\
\text { ciones). Categorías de respuesta: Nunca, 0/ A veces, } 1 \text { / } \\
\text { Siempre o muchas veces, } 2 \text {. }\end{array}$ & $\begin{array}{l}\text { Nivel [puntuación media/me- } \\
\text { diana/ desviación típica de una } \\
\text { escala de (1-2)]; prevalencia } \\
\text { [expuestos a cualquier nivel de } \\
\text { exposición }(\%) \text { ] }\end{array}$ \\
\hline $\begin{array}{l}\text { Trabajo } \\
\text { sedentario }\end{array}$ & $\begin{array}{l}\text { Realizar un trabajo sedentario. La evaluación se realiza en } \\
\text { base a la respuesta a un ítem: Su trabajo implica: Estar sen- } \\
\text { tado. Categorías de respuesta: Nunca, 0/ A veces, } 1 \text { / Siempre } \\
\text { o muchas veces, } 2 \text {. }\end{array}$ & $\begin{array}{l}\text { Nivel [puntuación media/me- } \\
\text { diana/ desviación típica de una } \\
\text { escala de }(1-2) \text { '; prevalencia } \\
\text { 'expuestos a cualquier nivel de } \\
\text { exposición }(\%) \text { ] }\end{array}$ \\
\hline Trabajar de pie & $\begin{array}{l}\text { Trabajar de pie. La evaluación se realiza en base a la res- } \\
\text { puesta a un ítem: Su trabajo implica: Estar de pie sin mover- } \\
\text { se. Categorías de respuesta: Nunca, 0/ A veces, } 1 / \text { Siempre o } \\
\text { muchas veces, } 2 \text {. }\end{array}$ & $\begin{array}{l}\text { Nivel [puntuación media/me- } \\
\text { diana/ desviación típica de una } \\
\text { escala de (1-2)]; prevalencia } \\
\text { [expuestos a cualquier nivel de } \\
\text { exposición }(\%) \text { ] }\end{array}$ \\
\hline Vibraciones & $\begin{array}{l}\text { Vibraciones de mano-brazo o cuerpo entero. La evaluación } \\
\text { se realiza en base a la respuesta a un ítem: ¿Está expuesto a: } \\
\text { Vibraciones (por herramientas manuales, maquinaria, etc.)? } \\
\text { Categorías de respuesta: Nunca, 0/ A veces, } 1 / \text { Siempre o } \\
\text { muchas veces, } 2 \text {. }\end{array}$ & $\begin{array}{l}\text { Nivel [puntuación media/me- } \\
\text { diana/ desviación típica de una } \\
\text { escala de }(1-2) \text { ]; prevalencia } \\
\text { [expuestos a cualquier nivel de } \\
\text { exposición }(\%) \text { ] }\end{array}$ \\
\hline $\begin{array}{l}\text { Pantallas de } \\
\text { visualización } \\
\text { de datos }\end{array}$ & $\begin{array}{l}\text { Utilización de pantallas de visualización de datos. La eva- } \\
\text { luación se realiza en base a la respuesta a un ítem: Su traba- } \\
\text { jo habitual implica utilizar: Equipos informáticos con tecla- } \\
\text { dos. Categorías de respuesta (Nunca, } 0 \text { / A veces, } 1 \text { / Siempre } \\
\text { o muchas veces, 2). }\end{array}$ & $\begin{array}{l}\text { Nivel [puntuación media/me- } \\
\text { diana/ desviación típica de una } \\
\text { escala de }(1-2) \text { ]; prevalencia } \\
\text { [expuestos a cualquier nivel de } \\
\text { exposición }(\%) \text { ] }\end{array}$ \\
\hline
\end{tabular}


Clasificación Nacional de Ocupaciones 1994 (CNO-94) ${ }^{6}$, según disponibilidad en las distintas fuentes utilizadas, buscando siempre el máximo nivel de desagregación posible (a mayor número de dígitos mayor especificidad en la definición de las ocupaciones).

En el presente análisis se describió la prevalencia de exposición a riesgos ergonómicos en población laboral española en los años 1997, 1999 y 2003. Se identificaron también las cinco ocupaciones con mayor prevalencia de exposición a dichos riesgos en el último año cubierto por MatEmESp (2005). Además se analizó la relación entre la prevalencia de exposición a los cuatro riesgos laborales asociados a la carga física con mayor prevalencia de exposición en 2005 (manipulación de cargas, posturas forzadas, movimientos repetidos y trabajo sedentario) y las características sociodemográficas (edad, sexo, nivel de estudios, país de origen y clase social) y laborales (situación de empleo, contrato temporal, trabajo a turnos, horas semanales y salario), para ocupaciones con tres dígitos según la CNO-94. Este análisis también se realizó con los datos del año 2005, a excepción de la relación entre salario (año 2006) y la exposición a riesgos ergonómicos, que se realizó para las ocupaciones con dos dígitos contenidas en la Encuesta Nacional de Condiciones de Trabajo (ENCT) del año 2003, en función de la información disponible en MatEmESp (por tanto, según la disponibilidad de esta información en las fuentes que alimentaron la matriz). Se utilizó el coeficiente de correlación de Spearman para valorar las relaciones debido a la ausencia de normalidad en la mayoría de las variables de estudio. Para explorar la relación entre las variables se realizaron gráficos de dispersión donde se representaron curvas suavizadas obtenidas mediante el método Loess. Los datos se analizaron con el paquete estadístico SPSS versión 19.

\section{RESULTADOS}

En la tabla 3 se describe la prevalencia de exposición a riesgos ergonómicos en población laboral española durante el período 1997-2003. Las exposiciones declaradas con mayor frecuencia fueron movimientos repetidos, afectando al $63 \%$ de los trabajadores españoles en 1997 y descendiendo al $60 \%$ en 2003 , posturas forzadas (23\% en 1997 llegando hasta el $29 \%$ en $2003)$ y manipulación de cargas $(29 \%$ en 1997 hasta el 36\% en 2003). En este período destacó el aumento en la exposición a posturas forzadas (23\% vs 29\%), manipulación de cargas $(29 \%$ vs. $36 \%)$, esfuerzo físico (24\% vs 31\%). También aumentó la exposición a trabajo sedentario, aunque de forma más moderada, (14\% vs 16\%), vibraciones $(7,8 \%$ vs $8,2 \%)$ y pantallas de visualización de datos (31\% vs 36\%), mientras que disminuyó ligeramente la prevalencia de trabajadores expuestos a movimientos repetidos (63\% vs. $60 \%)$ y bipedestación (13\% vs. 11,0\%).

En el último año con datos disponibles en MatEmESp (2005) la prevalencia de exposición a posturas forzadas en pobla-

Tabla 3

Prevalencias (\%) de exposición a riesgos ergonómicos en población trabajadora española, 1997-2003. Fuente MatEmESp (Matriz Empleo-Exposición Española: www.matemesp.org)

\begin{tabular}{|l|c|c|c|}
\hline & 1997 & 1999 & 2003 \\
\hline Posturas forzadas & 23,4 & 25,5 & 29,0 \\
\hline Manipulación de cargas & 28,8 & 32,2 & 35,6 \\
\hline Esfuerzo físico & 23,8 & 27,5 & 31,4 \\
\hline Movimientos repetitivos & 62,6 & 61,0 & 59,6 \\
\hline Trabajo sedentario & 13,6 & 14,2 & 15,6 \\
\hline Trabajo de pie & 12,5 & 13,6 & 11,0 \\
\hline Vibraciones & 7,8 & 7,2 & 8,2 \\
\hline PVD* & 31,4 & 29,4 & 35,7 \\
\hline
\end{tabular}

PVD*:Pantallas de visualización de datos 
ción laboral fue del 50\%, a manipulación de cargas del $36 \%$, a movimientos repetidos el $63 \%$ y a trabajo sedentario del $46 \%$.

La prevalencia de exposición a posturas forzadas en las ocupaciones (CNO-94 con tres dígitos) osciló entre el 9\% y $96 \%$, en manipulación de cargas entre el $0 \%$ y el $89 \%$, en movimientos repetidos entre el $15 \%$ y el $95 \%$ y en trabajo sedentario entre el $2 \%$ y el $100 \%$. La tabla 4 muestra las cinco ocupaciones con mayores prevalencias de exposición a los riesgos ergonómicos seleccionados en 2005. En la categoría de "albañiles y mamposteros" (CNO-94 711) fue donde se presentó un mayor número de riesgos ergonómicos con alta prevalencia de exposición: posturas forzadas $(96 \%)$, manipulación de cargas (79\%) y movimientos repetidos $(89 \%)$. Los "trabajadores de artes gráficas" (CNO-94 772) también estuvieron expuestos con mucha frecuencia a dos de estos riesgos, manipulación de cargas $(75 \%)$ y movimientos repetidos $(95 \%)$. Los "peones de construcción" (CNO-94 960) fueron los que presentaron la prevalencia de exposición más alta a manipulación de cargas $(89 \%)$. Las mayores prevalencias de exposición a trabajo sedentario se concentraron en ocupaciones relacionadas con trabajos de gestión administrativa con diferentes niveles de cualificación, "auxiliares administrativos" (CNO-94 430) con una prevalencia del 98\%, y "profesionales de apoyo en gestión financiera" (CNO-94 331), con el 97\% y en "conductores de automóviles y camiones" (CNO-94 861), afectando a la totalidad de los trabajadores en esta ocupación. Los "trabajadores de peluquería" (CNO-94 513) también presentaron una alta prevalencia de exposición a movimientos repetidos (91\%) y los "auxiliares de enfermería" (CNO-94 331) a manipulación de cargas $(75 \%)$.

En la tabla 5 se presentan los estimadores estadísticos que describen las relaciones entre la proporción de trabajadores expuestos (prevalencia de exposición) a los distin- tos riesgos considerados y la distribución de los trabajadores por condiciones de empleo y características sociodemográficas en cada ocupación. Según este análisis, la prevalencia de exposición a los riesgos ergonómicos evaluados en una determinada ocupación se relacionó con la proporción de trabajadores que no superaron el nivel de estudios primarios en esa ocupación. Conforme aumentaba la proporción de trabajadores de estudios primarios, crecía la prevalencia de exposición a posturas forzadas (coeficiente de correlación $=0,62$; $\mathrm{p}=<0,001$ ), manipulación de cargas (coeficiente de correlación $=0,59 ; \mathrm{p}=<0,001)$ y movimientos repetidos y viceversa (coeficiente de correlación $=0,66 ; p=<0,001)$. Por otro lado, conforme se incrementó la prevalencia de exposición a trabajo sedentario, disminuyó la proporción de ocupados con estudios primarios (coeficiente de correlación $=-0,62, p<0,001)$. Los niveles inferiores de clase social también se relacionaron con una mayor prevalencia de exposición a todos los riesgos ergonómicos evaluados: posturas forzadas (coeficiente de correlación $=0,61 ; p=<0,001)$, manipulación de cargas (coeficiente de correlación $=0,60$; $\mathrm{p}=<0,001$ ) y movimientos repetidos (coeficiente de correlación $=0,64 ; \mathrm{p}=<0,001)$ a excepción del trabajo sedentario, en el que al incrementar la proporción de trabajadores de clases sociales inferiores disminuyó la prevalencia de exposición (coeficiente de correlación $=-0,57, \mathrm{p}<0,001)$. También encontramos correlaciones superiores a 0,40 entre la proporción de hombres y de contratos temporales en una ocupación y la prevalencia de exposición a posturas forzadas (coeficientes de correlación $=0,44 \mathrm{y}$ 0,$43 ; p=0,002$ y 0,003 , respectivamente) y manipulación de cargas (coeficientes de correlación $=0,47$ y 0,$43 ; p=0,001$ y 0,003 respectivamente) en esa misma ocupación, así como entre la proporción de trabajadores españoles (no extranjeros) en una ocupación y la prevalencia de exposición a trabajo sedentario en la misma (coeficiente de correlación $=0,49, \mathrm{p}=0,001)$. 
Tabla 4

Ocupaciones con mayores prevalencias (\%) de trabajadores expuestos a riesgos ergonómicos, 2005. Fuente: MatEmESp (Matriz Empleo-Exposición Española, www.matemesp.org)

\begin{tabular}{|c|c|c|c|c|c|c|c|c|c|c|}
\hline \multicolumn{2}{|r|}{ Ocupación (CNO-94) } & \multirow{2}{*}{$\mathrm{N}$} & \multicolumn{2}{|c|}{$\begin{array}{l}\text { Posturas } \\
\text { forzadas }\end{array}$} & \multicolumn{2}{|c|}{$\begin{array}{c}\text { Manipulación } \\
\text { de cargas }\end{array}$} & \multicolumn{2}{|c|}{$\begin{array}{l}\text { Movimientos } \\
\text { repetidos }\end{array}$} & \multicolumn{2}{|c|}{$\begin{array}{c}\text { Trabajo } \\
\text { sedentario }\end{array}$} \\
\hline Código & Definición de la ocupación & & $\%$ & $\mathrm{n}$ & $\%$ & $\mathrm{n}$ & $\%$ & $\mathrm{n}$ & $\%$ & $\mathrm{n}$ \\
\hline 331 & Profesionales de apoyo en operaciones financieras y algunas operaciones comerciales & 195.564 & & & & & & & 97,1 & 189.862 \\
\hline 341 & Profesionales de apoyo de la gestión administrativa, con tareas administrativas generales & 691.606 & & & & & & & 95,8 & 662.761 \\
\hline 401 & Auxiliares contables y financieros & 158.281 & & & & & & & 95,9 & 151.719 \\
\hline 430 & Auxiliares administrativos sin tareas de atención al público no clasificados anteriormente & 452.258 & & & & & & & 97,9 & 442.853 \\
\hline 511 & Auxiliares de enfermería y asimilados & 228.139 & & & 75,2 & 171.593 & & & & \\
\hline 513 & Peluqueros, especialistas en tratamiento de belleza y trabajadores asimilados & 167.587 & & & & & 90,7 & 151.985 & & \\
\hline 711 & Albañiles y mamposteros & 627.386 & 95,6 & 599.781 & 79,1 & 496.358 & 88,6 & 556.033 & & \\
\hline 722 & Fontaneros e instaladores de tuberías & 152.357 & 88,9 & 135.457 & & & & & & \\
\hline 723 & Electricista de construcción y asimilados & 161.474 & 89,5 & 144.583 & & & & & & \\
\hline 729 & Otros trabajadores de acabado de construcción y asimilados & 118.508 & 89,6 & 106.228 & 76,3 & 90.453 & & & & \\
\hline 751 & Moldeadores, soldadores, chapistas, montadores de estructuras metálicas y trabajadores asimilados & 257.474 & & & & & & & & \\
\hline 772 & Trabajadores de artes gráficas y asimilados & 57.199 & & & 75,2 & 42.993 & 95,2 & 54.446 & & \\
\hline 831 & Operadores de máquinas para trabajar metales y otros productos minerales & 149.384 & & & & & 92,1 & 137.554 & & \\
\hline 833 & Operadores de máquinas para fabricar productos de caucho y plástico & 64.272 & 84,8 & 54.506 & & & & & & \\
\hline 836 & Operadores de máquinas para fabricar productos textiles artículos de piel y de cuero & 114.409 & & & & & 90,5 & 103.504 & & \\
\hline 861 & Taxistas y conductores de automóviles y furgonetas & 234.778 & & & & & & & 100,0 & 234.778 \\
\hline 960 & Peones de la construcción & 417.506 & & & 89,0 & 371.533 & & & & \\
\hline
\end{tabular}

N: número de trabajadores en la correspondiente ocupación; \%: prevalencia de exposición a cada riesgo ergonómico por ocupación; n: número de trabajadores expuestos, dato basado en la prevalencia de exposición y en el número de trabajadores por ocupación. 


\section{Tabla 5}

Relaciones (coeficiente de correlación) entre la distribución de trabajadores por ocupaciones según características personales y laborales y la prevalencia de exposición a riesgos ergonómicos en las distintas ocupaciones, 2005. Fuente: MatEmESp (Matriz Empleo-Exposición Española, www.matemesp.org)

\begin{tabular}{|c|c|c|c|c|c|c|c|c|}
\hline & \multicolumn{2}{|c|}{$\begin{array}{l}\text { Posturas } \\
\text { forzadas }\end{array}$} & \multicolumn{2}{|c|}{$\begin{array}{l}\text { Manipulación } \\
\text { de cargas }\end{array}$} & \multicolumn{2}{|c|}{$\begin{array}{l}\text { Movimientos } \\
\text { repetidos }\end{array}$} & \multicolumn{2}{|c|}{$\begin{array}{c}\text { Trabajo } \\
\text { sedentario }\end{array}$} \\
\hline & $\begin{array}{c}\text { coeficiente } \\
\text { de } \\
\text { correlación }\end{array}$ & $\mathrm{p}$ & $\begin{array}{c}\text { coeficiente } \\
\text { de } \\
\text { correlación }\end{array}$ & $\mathrm{p}$ & $\begin{array}{c}\text { coeficiente } \\
\text { de } \\
\text { correlación }\end{array}$ & $\mathrm{p}$ & $\begin{array}{l}\text { coeficiente } \\
\text { de } \\
\text { correlación }\end{array}$ & $\mathrm{p}$ \\
\hline Mujeres* & $-0,44$ & 0,002 & $-0,47$ & 0,001 & $-0,30$ & 0,041 & 0,28 & 0,058 \\
\hline \multicolumn{9}{|l|}{ Edad $^{* *}$} \\
\hline$<31$ & 0,08 & 0,605 & 0,12 & 0,423 & 0,19 & 0,205 & $-0,23$ & 0,130 \\
\hline$>45$ & 0,23 & 0,132 & 0,16 & 0,297 & 0,04 & 0,789 & $-0,01$ & 0,937 \\
\hline \multicolumn{9}{|l|}{ Nivel de estudios } \\
\hline Sin estudios & 0,42 & 0,003 & 0,45 & 0,002 & 0,61 & $<0,001$ & $-0,46$ & 0,001 \\
\hline Primarios & 0,62 & $<0,001$ & 0,59 & $<0,001$ & 0,66 & $<0,001$ & $-0,62$ & $<0,001$ \\
\hline Secundarios & 0,17 & 0,247 & 0,20 & 0,180 & 0,12 & 0,429 & $-0,26$ & 0,080 \\
\hline Universitarios & $-0,65$ & $<0,001$ & $-0,54$ & $<0,001$ & $-0,65$ & $<0,001$ & 0,55 & $<0,001$ \\
\hline Origen extranjero* & 0,36 & 0,015 & 0,35 & 0,016 & 0,30 & 0,043 & $-0,49$ & 0,001 \\
\hline Clase Social (I-V) & 0,61 & $<0,001$ & 0,60 & $<0,001$ & 0,64 & $<0,001$ & $-0,57$ & $<0,001$ \\
\hline \multicolumn{9}{|l|}{ Situación de empleo } \\
\hline Asalariados & 0,07 & 0,643 & 0,12 & 0,410 & $-0,02$ & 0,885 & 0,01 & 0,939 \\
\hline Autónomos & 0,05 & 0,740 & $-0,05$ & 0,738 & 0,10 & 0,491 & $-0,04$ & 0,793 \\
\hline Empleadores & $-0,02$ & 0,904 & $-0,10$ & 0,503 & $-0,04$ & 0,774 & $-0,07$ & 0,643 \\
\hline Contrato temporal & 0,42 & 0,004 & 0,39 & 0,008 & 0,30 & 0,041 & $-0,43$ & 0,003 \\
\hline Horas semanales & 0,24 & 0,113 & 0,27 & 0,071 & 0,23 & 0,128 & $-0,21$ & 0,167 \\
\hline Salario bruto & $-0,27$ & 0,066 & $-0,37$ & 0,011 & $-0,39$ & 0,007 & 0,26 & 0,080 \\
\hline
\end{tabular}

* Se obtiene el mismo coeficiente de correlación (con signo contrario) y p en la correlación entre las variables de riesgos ergonómicos y la variable complementaria (proporción de hombres o proporción de personas de origen español). Se omite en la tabla para no mostrar contenido redundante.

** Sólo se muestra la correlación con los grupos de edad extremos.

En la figura 1 se representa la relación entre la exposición a riesgos ergonómicos y algunas variables sociodemográficas seleccionadas. Así, aunque el coeficiente de correlación entre la proporción de mujeres en una ocupación y la prevalencia de posturas forzadas y manipulación de cargas fue negativo, es decir las ocupaciones menos feminizadas son las que mayor frecuencia de exposición presentaban (coeficientes de correlación $=-0,44$ y $-0,47 ; p=0,002$ y 0,001 , respectivamente), algunas ocupacio- nes muy feminizadas, como auxiliares de enfermería (CNO-94 511), también mostraron prevalencias elevadas a este tipo de riesgos $(69 \%$ y $75 \%$ ) (figura 1a y $1 \mathrm{~b})$. Del mismo modo, aunque el coeficiente de correlación entre la proporción de mujeres y la exposición a trabajo sedentario fue positiva, aunque no estadísticamente significativa, (coeficiente de correlación $=0,28 ; p=0,058$ ), ocupaciones muy masculinizadas, como "conductores de automóviles" (CNO-94 861) también mostraron una alta prevalen- 


\section{Figura 1}

Relaciones entre la distribución de trabajadores por ocupaciones según características personales y la prevalencia de exposición a riesgos ergonómicos en las distintas ocupaciones, 2005. Fuente: MatEmESp

(Matriz Empleo-Exposición Española, www.matemesp.org).

Figura 1a

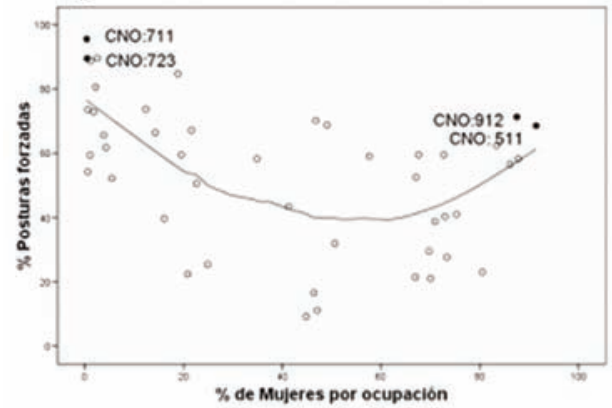

Figura 1c

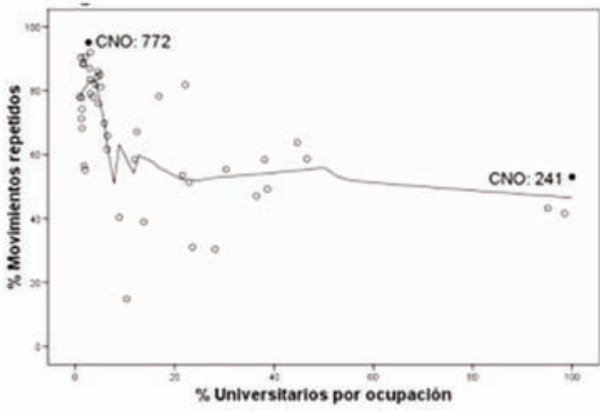

Figura $1 \mathrm{~b}$

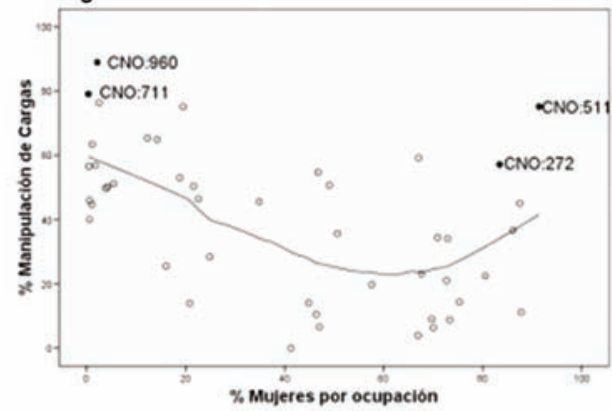

Figura 1d

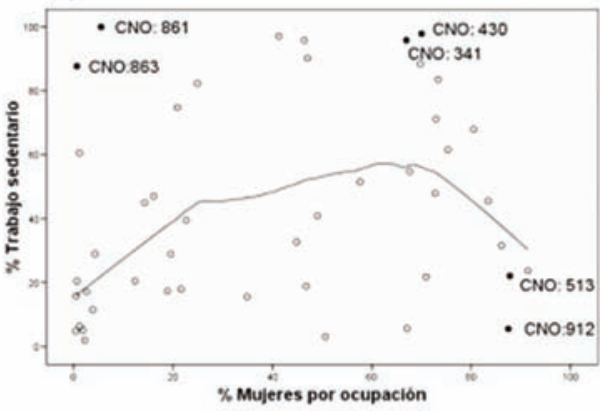

CNO (Código Nacional de Ocupaciones-94) 241: profesionales en organización y administración de empresas; CNO 272: personal de enfermería; CNO 341: profesionales de apoyo de la gestión administrativa; CNO 430: auxiliares administrativos sin tareas de atención al público; CNO 511: auxiliares de enfermería; CNO 513: peluqueros y trabajadores asimilados; CNO 711: albañiles y mamposteros; CNO 723: electricista de construcción y asimilados; CNO 772: trabajadores de artes gráficas y asimilados; CNO 861: taxistas y conductores de automóviles y furgonetas; CNO 863: conductores de camiones; CNO 912: personal de limpieza de oficinas y otros establecimientos similares; CNO 960: peones de la construcción.

cia de exposición a este riesgo (100\%) y ocupaciones muy feminizadas, como "personal de limpieza" (CNO-94 912) mostraron baja prevalencia de exposición $(6 \%)$ (figura 1d). Por último, aunque el coeficiente de correlación entre la proporción de trabajadores con nivel de estudios universitario y la prevalencia de movimientos repetidos fue negativo (coeficiente de correlación $=-0,65 ; \mathrm{p}=<0,001)$, ocupaciones con alta proporción de universitarios, como "profesionales de administración de empresas", también estaban expuestos con mucha frecuencia a este riesgo (53\%) (figura 1c).

\section{DISCUSIÓN}

La prevalencia de exposición laboral a algunos de los principales riesgos ergonómicos en población trabajadora en España fue en aumento desde 1997 a 2003. En gene- 
ral, las ocupaciones más expuestas a riesgos ergonómicos, a excepción de trabajo sedentario, se relacionan con trabajos manuales de construcción, industria y servicios. También se encuentra una alta prevalencia de trabajo sedentario en trabajadores no manuales de gestión administrativa. El trabajo sedentario es más frecuente en ocupaciones con mayor proporción de trabajadores con nivel de estudios superiores, mayor proporción de autóctonos y menor proporción de contratos temporales. Para la mayoría de los restantes riesgos ergonómicos considerados (posturas forzadas, manipulación de cargas y movimientos repetidos) la exposición es más frecuente en ocupaciones en las que trabajan con mayor frecuencia personas de clases sociales más desfavorecidas, hombres, personas con estudios primarios, inmigrantes (personas de origen no español) y trabajadores con contratos temporales y con menor salario bruto. Sin embargo, encontramos excepciones a este patrón. Algunas ocupaciones más feminizadas y ocupaciones en las que la proporción de trabajadores con alto nivel de estudios es elevada, también presentan prevalencias altas de exposición a determinados riesgos ergonómicos por ejemplo las "enfermeras" y "auxiliares de enfermería" presentan alta prevalencia de exposición a manipulación de cargas.

La exposición a riesgos ergonómicos en el trabajo es causa de fatiga crónica y trastornos musculoesqueléticos (TME) de diversa índole ${ }^{21}$. Los TME son los problemas de salud relacionados con el trabajo con mayor frecuencia referidos por los trabajadores europeos y también en España ${ }^{22,23}$. Los TME relacionados con la carga física en el trabajo en 2012 fueron también la primera causa de enfermedad profesional registrada en España ${ }^{24}$ y en 2011 los accidentes por sobreesfuerzos fueron los más frecuentes de los accidentes con baja laboral (38\%), causando el 34\% de las jornadas perdidas ${ }^{25}$. En España, en 2005, el 76\% de los accidentes producidos por sobreesfuerzo ${ }^{25}$ se pre- sentaron en hombres, dato coherente con la correlación inversa observada en nuestro análisis entre la prevalencia de exposición a manipulación de cargas y la proporción de mujeres en las ocupaciones.

Todo nuestro análisis se ha basado en la información contenida en MatEmESp y, por tanto, comparte las limitaciones que tiene esta herramienta, que a su vez tiene también las limitaciones de las fuentes que han proporcionado los datos a la matriz. Así, MatEmESp no dispone de información sobre la exposición para todas las ocupaciones incluidas en la CNO-94, sin embargo, hemos comprobado que las ocupaciones no incluidas (con dos dígitos) representaban menos del $1 \%$ del total de personas ocupadas en el año 2005. Por otra parte, el período temporal cubierto por MatEmESp se limita a 1996-2005. Aunque las enormes variaciones en el mercado laboral y en la estructura productiva ocurridas en nuestro país desde entonces han modificado el número y la distribución de trabajadores en las distintas ocupaciones ${ }^{26}$, en principio estas diferencias no tendrían por qué haber influido en las relaciones internas entre las tareas propias de cada ocupación y las consecuentes exposiciones laborales derivadas de dichas tareas, sin embargo esto es algo que no podemos comprobar con nuestros datos. Para tener esa perspectiva temporal sería necesario actualizar periódicamente la información en MatEmESp, algo que, a nuestro entender, debería ser del interés de las autoridades sanitarias y laborales y deberían plantearse seriamente. En países como Finlandia, un organismo oficial, el Finnish Institute for Occupational Health, es el que asume el mantenimiento y revisión periódica de FINJEM $^{8}$, la matriz-empleo exposición finlandesa, tarea, por otra parte, facilitada por la riqueza y centralización de los registros de salud pública en este país (incluyendo la salud laboral).

Como ya hemos comentado, la calidad de los datos de MatEmESp, y por tanto de los 
resultados obtenidos con los análisis de la información contenida en la matriz, depende de la calidad de las fuentes originales con la que se ha abastecido la herramienta. Las estimaciones de exposición a riesgos ergonómicos en MatEmESp se basan en la información referida por los trabajadores en encuestas de condiciones de trabajo, cuya utilidad es incuestionable pero que en nuestro país no están exentas de problemas metodológicos ${ }^{27,28}$. También se conocen las limitaciones de la validez de la información sobre exposición laboral a carga física referida por los trabajadores frente a la obtenida mediante observación o evaluación de expertos $^{29}$. Sin embargo, la exposición autoreferida también presenta ventajas, como su facilidad de uso, el bajo coste y su mejor adecuación para evaluar un número elevado de puestos de trabajo en períodos prolongados, generando también menor rechazo en las personas evaluadas ${ }^{29,30}$.

La utilización de las ocupaciones codificadas en CNO-94 como unidades de observación conlleva igualmente alguna desventaja. La CNO es un sistema creado fundamentalmente con fines administrativos y los literales que describen algunas ocupaciones resultan en ocasiones insuficientes o inespecíficos. Sin embargo, la CNO-94 es un instrumento de clasificación uniforme que viene usándose de forma habitual en registros administrativos y encuestas oficiales en España. Además está basada en la Clasificación Internacional (CIUO-88), permitiendo la comparación de los datos de MatEmESp con los de otras matrices o estudios que utilicen la clasificación internacional.

Por último, cabría comentar que se han encontrado diferencias en los datos disponibles en MatEmESp sobre prevalencia de exposición a riesgos ergonómicos por ocupación, que no son atribuibles a variaciones en las condiciones de trabajo sino a la aplicación de diferentes criterios en las fuentes de las que se obtienen los datos para la construcción de la matriz. En concreto, hemos comprobado que existen diferencias sustanciales en la información derivada de la III Encuesta Nacional de Condiciones de Trabajo (2003) y la I Encuesta Catalana de Condiciones de Trabajo (2005) que son difícilmente atribuibles a diferencias relacionadas con el ámbito y/o periodo de ambas encuestas sino a diferencias en las correspondientes preguntas y opciones de respuesta en las encuestas (tablas 1 y 2). En MatEmESp, y por tanto en este análisis, se han considerado datos procedentes de distintas fuentes. Por tanto, aunque a nuestro entender esta información sigue siendo útil para categorizar y/o establecer relaciones ordinales entre las ocupaciones (por ejemplo, en cuanto a la frecuencia relativa de trabajadores expuestos a los diferentes riesgos), su utilización para cuantificar el número de trabajadores expuestos a los riesgos correspondientes en cada ocupación debe ser más prudente. Las variaciones en los criterios de evaluación de riesgos laborales que siguen las diferentes encuestas de condiciones de trabajo explican las dificultades encontradas a la hora de comparar los resultados de estas encuestas ${ }^{31}$.

Con las precauciones previamente señaladas, el análisis presentado en este trabajo permite identificar las ocupaciones con las prevalencias más altas de trabajadores expuestos a los principales riesgos ergonómicos. Con los datos disponibles, también es posible describir, a nivel de ocupación, desigualdades en las situaciones de exposición en función de características personales y de empleo en los trabajadores. MatEmESp también dispone de información sobre el nivel de exposición a riesgos ergonómicos en algunas ocupaciones según sexo y edad. Sería interesante profundizar en esta información contenida en la matriz para analizar las desigualdades por estas variables presentes en una misma ocupación, desigualdades que pueden ser debidas a diferencias en las tareas desempeñadas en la misma ocupación según características personales de los trabajadores o a las diferentes activi- 
dades industriales donde pueden encontrarse estas ocupaciones segregadas por sexo y/o edad ${ }^{32}$. Esta información sin duda puede llevar a una mejora en la aplicación de medidas preventivas al localizar ocupaciones y colectivos específicos con mayores riesgos en función de la prevalencia de exposición y no tanto en la incidencia de daños a la salud.

Los datos obtenidos en esta explotación de MatEmESp llevan a la conclusión de que es necesario prestar mayor atención a la prevención de riesgos ergonómicos en la población trabajadora española, haciendo especial hincapié en trabajadores manuales, donde se concentran los trabajadores con características personales y laborales más desfavorecidas, pero sin olvidarse de algunas ocupaciones más cualificadas que también presentan alta exposición a estos riesgos.

\section{BIBLIOGRAFÍA}

1. Kauppinen TP. Assessment of exposure in occupational epidemiology. Scand J Work Environ Health. 1994;20 Spec Issue:19-29.

2. Alguacil J, Kauppinen T, Porta M, Partanen T, Malats N, Kogevinas M, et al. Risk of pancreatic cancer and occupational exposures in Spain. PANKRAS II Study Group. Ann Occup Hyg. 2000;44(5):391-403.

3. Santibañez M, Alguacil J, García de la Hera M, Navarrete-Muñoz EM, Llorca J, Aragonés N, et al. Occupational exposures and risk of stomach cancer by histological type. Occup Environ Med. 2012;69(4):268-75.

4. Bosch de Basea M, Porta M, Alguacil J, Puigdomènech E, Gasull M, Garrido JA, et al. Relationships between occupational history and serum concentrations of organochlorine compounds in exocrine pancreatic cancer. Occup Environ Med. 2011;68(5):332-8.

5. Kromhout H, Vermeulen R. Application of job-exposurre matrices in studies of general population: some clues to their performance. Eur Respir Rev. 2001;11(80):80-90.

6. Instituto Nacional de Estadística. Clasificación Nacional de Ocupaciones (CNO-94). Disponible en: http://www.ine.es
7. García A, González-Galarzo M, Kauppinen T, Delclos G, Benavides F. A Job-Exposure Matrix for Research and Surveillance of Occupational Health and Safety in Spanish Workers: MatEmESp. Am J Ind Med. 2013;56(10):1226-38.

8. Kauppinen T, Toikkanen J, Pukkala E. From cross-tabulations to multipurpose exposure information systems: a new job-exposure matrix. Am J Ind Med. 1998;33(4):409-17.

9. Blanc PD, Faucett J, Kennedy JJ, Cisternas M, Yelin E. Self-reported carpal tunnel syndrome: Predictors of work disability from the National Health Interview Survey Occupational Health Supplement. Am J Ind Med. 1996;30(3):362-8.

10. Svendsen SW, Johnsen B, Fuglsang-Frederiksen A, Frost P. Ulnar neuropathy and ulnar neuropathylike symptoms in relation to biomechanical exposures assessed by a job exposure matrix: a triple case-referent study. Occup Environ Med. 2012;69(11):773-80.

11. Johnson JV, Stewart WF. Measuring work organization exposure over the life course with a jobexposure matrix. Scand J Work Environ Health. $1993 ; 19(1): 21-8$.

12. Village J, Koehoorn M, Hossain S, Ostry A. Quantifying tasks, ergonomic exposures and injury rates among school custodial workers. Ergonomics. 2009;52(6):723-34.

13. Boyer J, Galizzi M, Cifuentes M, d' Errico A, Gore R, Punnett L, et al. Ergonomic and socioeconomic risk factors for hospital workers' compensation injury claims. Am J Ind Med. 2009;52(7):55162.

14. Maher N. The application of a job exposure matrix in the natural gas industry. AIHA J (Fairfax, Va). 2003;64(6):806-14.

15. US Department of Labor/Employment and Training Administration. O’NET Resorce Center. Disponible en: http://www.onetcenter.org/

16. Observatorio Estatal de Condiciones de Trabajo. Instituto Nacional de Seguridad e Higiene en el Trabajo. Ministerio de Trabajo e Inmigración. Encuestas de Condiciones de Trabajo y Gestión. Disponible en: http://www.oect.es

17. Direcció General de Relacions Laborals. Departament de Treball. Generalitat Catalana. Primera Enquesta de condicions de treball de Catalunya. Disponible en: http://www.gencat.es 
18. Almodóvar A, Pinilla FJ, Zimmermann M, Hervás P. Encuesta Nacional de Condiciones de Trabajo en el sector agropecuario. Disponible en: http://www.oect.es/Observatorio/Contenidos/InformesPropios/Desarrollados/Fich eros/Encuesta\%20Nacional\%20Agropecuaria.pdf

19. Instituto Nacional de Estadística. Clasificación Nacional de Actividades Económicas (CNAE-93-Rev.1). Disponible en: http://www.ine.es

20. Grupo de Trabajo de la Sociedad Española de Epidemiologia y de la Sociedad Española de Medicina de Familia y Comunitaria, Domingo-Salvany A, Regidor E, Alonso J, Alvarez-Dardet C. Una propuesta de medida de la clase social. Aten Primaria. 2000;25(5):350-63.

21. Ruiz-Frutos C, García AM, Delclos J, Benavides FG. Salud laboral. Conceptos y técnicas para la prevención de riesgos laborales. Barcelona: Elsevier-Masson; 2007.

22. European Foundation for the Improvement of Living and Working Conditions. Fourth European Working Conditions Survey. Disponible en: http://www.eurofound.europa.eu/pubdocs/2006/98/en/2/ ef0698en.pdf

23. Instituto Nacional de Seguridad e Higiene en el Trabajo. VI Encuesta Nacional de Condiciones de Trabajo. Disponible en: http://www.oect.es/portal/site/Observatorio/

24. Observatorio de Contingencias Profesionales y de la Seguridad Social. Enfermedades Profesionales (CEPROSS). Disponible en: http://www.segsocial.es/Internet_1/Estadistica/Est/Observatorio_de_las _Enfermedades_Profesionales/index.htm

25. Ministerio de Empleo y Seguridad Social. Estadística de Accidentes de trabajo. Disponible en: http://www.empleo.gob.es/estadisticas/eat/welcome.htm

26. Instituto Nacional de Estadística. Encuesta de Población Activa. Disponible en: http://www.ine.es

27. Artazcoz L. Encuesta sobre condiciones del trabajo: Reflexiones y sugerencias. Arch Prev Riesgos Labor. 2003;6(1):1-3.

28. Campos J, Almodóvar A, Pinilla J, Benavides F. Recomendaciones metodológicas para el diseño de encuestas de condiciones de trabajo y salud. Arch Prev Riesgos Labor. 2009;12(4):195-198.

29. Barriera-Viruet H, Sobeih TM, Daraiseh N, Salem S. Questionnaires vs observational and direct measurements: a systematic review. Theor Issues Ergon. 2006;7(3):261-84.
30. David GC. Ergonomic methods for assessing exposure to risk factors for work-related musculoskeletal disorders. Occup Med (Lond). 2005;55(3):190-9.

31. Narocki C, Zimmermann M, Artazcoz L, Gimeno D, Benavides FG. Encuesta de condiciones de trabajo y salud en España: comparación de los contenidos del cuestionario del trabajador. Arch Prev Riesgos Labor. 2009;12(2):60-8.

32. Eng A, 't Mannetje A, McLean D, Ellison-Loschmann L, Cheng S, Pearce N. Gender differences in occupational exposure patterns. Occup Environ Med. 2011;68(12):888-94. 